\title{
A Mechanistic Model for Depth-Dependent Hardness of Ion Irradiated Metals
}

\author{
Xiazi Xiao ${ }^{1,2,3}$, Qianying Chen ${ }^{1,2}$, Hui Yang ${ }^{3}$, Huiling Duan ${ }^{1,2^{*}}$ and Jianmin $\mathrm{Qu}^{3^{*}}$ \\ ${ }^{1}$ State Key Laboratory for Turbulence and Complex System, Department of Mechanics and \\ Engineering Science, College of Engineering, Peking University, Beijing 100871, P.R. China \\ ${ }^{2}$ CAPT, HEDPS and IFSA Collaborative Innovation Center of MoE, BIC-ESAT, Peking \\ University, Beijing 100871, P.R. China \\ ${ }^{3}$ School of Engineering, Tufts University, Medford, MA 02155, USA
}

\begin{abstract}
A mechanistic model was developed for modeling the depth-dependent hardness in ion irradiated metallic materials. The model is capable of capturing the indentation size effect, ion irradiation induced damage gradient effect, and effect of unirradiated region acting as a soft substrate. A procedure was developed and described in detail to parametrize the model based on experimentally obtained hardness vs. indentation depth curves. Very good agreement was observed between our model predictions and experimental data of several different stainless steels subjected to various ion irradiation conditions. In addition, two hardening mechanisms are revealed in the new model. One is the well-known indentation size effect arising from the creation of geometrically necessary dislocations as the indenter pierces into the materials. The other is the irradiation hardening due to the presence of irradiation-induced defects. As a function of indentation depth $h$, the hardening due to indentation size effect is described by $\bar{h}^{*} / h$, while the hardening due to irradiation first follows a power law form $P h^{n}$, then changes to $Z / h-Q / h^{3}$, where $\bar{h}^{*}, P, n, Z$ and $Q>0$ are constants. This transition occurs at the indentation depth when the plastic zone reaches the end of the irradiated layer.
\end{abstract}

Keywords: Hardness, Irradiation effect, Theoretical model, Finite element modeling, Stainless steel

*Corresponding authors. E-mails:hlduan@pku.edu.cn; Jianmin.Qu@tufts.edu 


\section{Introduction}

Irradiation-induced damage is one of the major safety threats to the nuclear industry. Full understanding of irradiation-induced material degradation is vital not only to the development of new materials for the next generation nuclear reactors, but also to the extension of the operating lifetimes of the current fleet of nuclear reactors.

Studying the mechanical behavior of metallic materials subjected to neutron irradiation has long been a challenging problem due to the limited availability of neutron sources, high radioactivity of irradiated samples, and long experimental period and high cost. As an alternative, ion irradiated samples have often been used as surrogates to study irradiation damage in metallic materials, because ion irradiation not only induces defects that are similar to those induced by neutron irradiation [1-7], but also has many advantages over neutron irradiation, namely, more availability of ion sources, low radioactivity of irradiated materials, reduced costs and irradiation times, controllable irradiation conditions and the possibility for co-implantation of various kinds of irradiation particles [8-10].

Compared to neutron irradiation, ion irradiation does have some limitations. Chief among them is that ion irradiation can only penetrate a very limited depth (a few micrometers) into the sample, and the resulting defect distribution within this irradiated layer is often non-uniform. This makes it almost impossible to fabricate irradiated samples for traditional mechanical tests such as torsional and tensile tests [8-12].

However, the fact that ion irradiation creates a thin surface layer with non-uniformly distributed defects makes ion irradiated samples ideally suited for indentation tests [13]. Through the continuous stiffness measurement (CSM) technique, the depth-dependent hardness can be directly obtained experimentally [14]. Once the hardness $H$ is measured, the material's yield strength $\sigma_{\mathrm{YS}}$ can be approximately related to its hardness through the empirical formula [7] $\sigma_{\mathrm{YS}}=H / k_{\mathrm{H}}$, where $k_{\mathrm{H}}$ is a proportionality coefficient. For most metals, $k_{\mathrm{H}} \approx 3$ [15].

In recent years, numbers of indentation tests have been performed to investigate the influence of ion irradiation on the hardness of metallic materials, including single crystals $[12,16]$, pure polycrystals [17, 18], and alloys [7, 8, 19-27]. Among them, stainless steels, a widely used material in nuclear reactors due to their excellent strength, ductility and corrosion resistance, are the most studied materials [5-7, 11, 28-32].

For example, Chen et al. [11] irradiated samples made of a solution annealed stainless steel 
with $3 \mathrm{MeV}$ ion at $673 \mathrm{~K}$ for up to 3 displacements per atom (dpa). They found that the irradiation-induced defects were primarily dislocation loops of similar size to each other, these defects were non-uniformly distributed in the irradiated region. Huang, et al. [7] irradiated samples made of $20 \%$ cold-worked 316 stainless steel with $7 \mathrm{MeV}$ ions. They also found that dislocation loops were the primary defects. Their average number density was about $3 \times$ $10^{22} \mathrm{~m}^{-3}$, and the loop size was a few nanometers. Yabuuchi, et al. [5] irradiated samples made of SUS316L stainless steel with $1 \mathrm{MeV}$ ions at $573 \mathrm{~K}$ with different irradiation doses, and measured the depth-dependent hardness.

These and other studies on various stainless steels subjected to a wide range of ion irradiation conditions seem to indicate that, similar to neutron irradiation [1-4], ion irradiation induces mainly dislocation loops in stainless steels [5-7]. Furthermore, these studies demonstrated that the material behavior under indentation tests shows similar trends: (1) Similar to indenting unirradiated materials, there is an indentation size effect in ion irradiated materials as well, wherein the hardness is observed to increase with decreasing indentation depth, especially in the sub-micrometer depth regime; (2) There is a damage gradient effect in that hardness generally increases with increasing density of irradiation-induced defects, and eventually reaches an upper limit with further increase of the irradiation dose; (3) There is a soft substrate effect, namely, there exists a threshold indentation depth beyond which the hardness vs. depth profile changes significantly, signaling the fact that the plastic zone induced by the indenter has extended deep into the unirradiated material. For stainless steels, this threshold value is about one-tenth to onefifth of the thickness of the irradiated layer.

Several models have been proposed to explain the experimental data, and to understand the fundamental mechanisms related to the depth-dependent hardness in ion irradiated materials [3237]. For instance, the Nix-Gao model $[33,34]$ has been used to explain the indentation size effect. The damage gradient effect is accounted for by the non-uniform distribution of irradiationinduced defects in refs. [36, 37], while the soft substrate effect is attributed to the competition between the non-uniform distribution of defect density in the irradiated layer and the unirradiated material acts as a soft substrate [35-37]. In spite of the extensive effort, these existing models still have serious shortcomings.

First of all, the Nix-Gao model [33] was originally developed for homogeneous materials. The depth-dependent hardness predicted by this model is entirely due to the presence of the 
geometrically necessary dislocations. In ion irradiated material, the irradiation-induced defect will add additional hardening that cannot be captured by using only the geometrically necessary dislocations. Even though some authors have used the Nix-Gao model to fit the experimentally obtained hardness vs. depth curves in ion irradiated materials [5, 24], the physical interpretation of the two fitting parameters $H_{0}$ and $h^{*}$ in the Nix-Gao model become questionable, because neither of them contains information about the irradiation-induced defects. Moreover, the NixGao model can only be approximately employed when the indentation-induced plastic zone is within the irradiated layer [28], beyond which the soft substrate effect becomes increasingly important.

Secondly, most of the existing models for irradiation hardening are based on the well-known Orowan model which, strictly speaking, is applicable only when the defect distribution is uniform. So, to apply the Orowan model to ion irradiated materials, where the defects are nonuniformly distributed, the average defect distribution may have to be used [8]. Note that irradiation hardening is primarily caused by the irradiation-induced defects within the plastic zone. When the plastic zone is fully contained within the irradiated layer, the defect density in the plastic zone is rather non-uniform, therefore, the Orowan model needs to reflect the nonuniform defect distribution in order to be applicable to ion irradiated materials with a nonuniform defect density profile [11].

In this paper, a new model is proposed for the depth-dependent hardness of ion irradiated metallic materials. The model is based on the deformation mechanisms during the indentation of ion irradiated materials. The model is capable of capturing the indentation size effect, the damage gradient effect, and the soft substrate effect. Detailed derivation of the model is presented in Section 2. A procedure to parametrize the model is described in Section 3. The parameterized model is then used in Section 4 to simulate the hardness vs. indentation depth curves obtained experimentally on three different stainless steels subjected to various irradiation conditions. Finally, some conclusion remarks are given in Section 5.

\section{Model Development}

During ion irradiation, defects such as dislocation loops and stacking fault tetrahedrons can be created. These irradiation-induced defects act as barriers for the motion of dislocations. Consequently, the yield strength of irradiated materials increases [8]. In general, such irradiation 
hardening depends on the density and distribution of irradiation-induced defects as well as the interaction between irradiation-induced defects and existing or newly generated dislocations in the plastically deformed region during the deformation process.

The yield strength of a material is related to the shear stress needed to move dislocations, or the critical resolved shear stress which, in general, can be written as

$$
\tau_{\mathrm{CRSS}} \propto \mu b \sqrt{\rho},
$$

where $\mu$ is the shear modulus, $b$ the magnitude of Burgers vector, $\rho$ the density of dislocation barriers. To make Eq. (1) an equality, a coefficient is often introduced in front of $\sqrt{\rho}$. This coefficient is commonly called the hardening coefficient. Its value represents the strength of the barrier in preventing the motion of dislocations. Thus, different types of barriers may have different hardening coefficients.

Let the density of dislocations be $\rho_{\text {dis }}$ before irradiation, and $N_{\text {def }}$ be the number density of the irradiation-induced defects, and their average size be $d_{\text {def }}$. Then Eq. (1) for the irradiated material can be written as

$$
\tau_{\mathrm{CRSS}}^{\mathrm{irr}}=\mu b \sqrt{\alpha^{2} \rho_{\mathrm{dis}}+\beta^{2} N_{\mathrm{def}} d_{\mathrm{def}}}
$$

where $\alpha$ is the hardening coefficient of dislocations, and $\beta$ is the hardening coefficient of defects induced by irradiation.

It is recognized immediately that $\Delta \tau_{\mathrm{CRSS}}^{\mathrm{dis}}=\mu b \alpha \sqrt{\rho_{\mathrm{dis}}}$ is the critical resolved shear stress of the unirradiated material, and $\Delta \tau_{\mathrm{CRSS}}^{\mathrm{def}}=\mu b \beta \sqrt{N_{\mathrm{def}} d_{\mathrm{def}}}$ may be considered as the critical resolved shear stress resulted from the additional defects generated by irradiation. In other words, Eq. (2) can be written as

$$
\tau_{\mathrm{CRSS}}^{\mathrm{irr}}=\sqrt{\left(\Delta \tau_{\mathrm{CRSS}}^{\mathrm{dis}}\right)^{2}+\left(\Delta \tau_{\mathrm{CRSS}}^{\mathrm{def}}\right)^{2}}
$$

This root-sum-square model was shown by Foreman and Makin [38] to apply for a population of barriers with similar strengths, i.e., $\Delta \tau_{\mathrm{CRSS}}^{\mathrm{dis}}$ and $\Delta \tau_{\mathrm{CRSS}}^{\mathrm{def}}$ are on the same order of magnitude. If either one is much larger than the other, it can be easily shown through a simple algebraic manipulation that the following linear superposition applies,

$$
\tau_{\mathrm{CRSS}}^{\mathrm{irr}}=\Delta \tau_{\mathrm{CRSS}}^{\mathrm{dis}}+\Delta \tau_{\mathrm{CRSS}}^{\mathrm{def}}
$$


As demonstrated by the examples discussed in this work, Eq. (3) yields better fitting results than Eq. (4) when compared with different sets of experimental data of ion irradiated materials, which include stainless steels [5, 30], China A508-3 steels [23] and 16MND5 steels [22] at different irradiation conditions.

Further, we note that in many practical applications not all the dislocations and defects in irradiated materials contribute to material hardening. For example, during an indentation test, only dislocation barriers within the plastic zone near the indenter contribute to the increased yield strength. Additionally, $\rho_{\text {dis }}$ and $N_{\text {def }}$ may not be uniform within the plastic zone. Therefore, for simplicity, the $\rho_{\text {dis }}$ and $N_{\text {def }}$ in Eq. (2) will be replaced in this work by their spatial averages over the indentation-induced plastic zone, $\bar{\rho}_{\text {dis }}=\left(1 / V_{\mathrm{p}}\right) \int_{V_{\mathrm{p}}} \rho_{\mathrm{dis}} \mathrm{dV}$ and $\bar{N}_{\text {def }}=\left(1 / V_{\mathrm{p}}\right) \int_{V_{\mathrm{p}}} N_{\text {def }} \mathrm{dV}$, respectively, where $V_{\mathrm{p}}$ is the plastic zone volume.

Since the hardness of a material can be related to its critical resolved shear stress through the Mises flow rule [39] and Tabor's factor [40], it then follows from Eq. (2) that the hardness of irradiated materials is given by

$$
H_{\mathrm{irr}}=3 \sqrt{3} \tau_{\mathrm{CRSS}}^{\mathrm{irr}}=H_{\mathrm{unirr}} \sqrt{1+\beta^{2} \bar{N}_{\mathrm{def}} d_{\mathrm{def}} /\left(\alpha^{2} \bar{\rho}_{\mathrm{dis}}\right)},
$$

where

$$
H_{\text {unirr }}=3 \sqrt{3} \mu b \alpha \sqrt{\bar{\rho}_{\text {dis }}}
$$

is the hardness of the unirradiated material. Eq. (5) indicates that the hardness of irradiated materials can be predicted once the quantities on the right-hand side of Eq. (5) are known.

In what follows, we will evaluate the terms on the right-hand side of Eq. (5) to develop a model for the depth-dependent hardness of ion irradiated materials obtained through indentation measurements. For the purpose of developing the model, we will use a conical-shaped indenter with $\theta$ being the base angle (complementary of the half apex angle). It is noted that other indenter shapes, such as Vickers and Berkovich, can be approximated by a conical indenter with the same projected area for any given depth [41]. We further introduce a coordinate $x$, which originates from the point on the sample surface that contacts with the indenter tip at the beginning of indentation, and points toward the inside of the sample being indented. Thus, according to Eq. (5), the depth-dependent hardness will come from the dependence of $\bar{\rho}_{\text {dis }}$ and 
$\bar{N}_{\text {def }}$ on $x$, more specifically, the dependence of $\rho_{\text {dis }}$ and $N_{\text {def }}$ on $x$.

First, let us consider $N_{\text {def }}(x)$. Since there exists a nearly linear relationship between the irradiation dose and the defect density generated by irradiation [42], one may take the $x$ dependence of $N_{\text {def }}(x)$ as that of the irradiation does, which, in most ion irradiation experiments, is found to firstly increase with irradiation depth, and then rapidly drops to zero after it reaches a peak value at the maximum irradiation depth $[5,30]$. Thus, for simplicity, we assume the following spatial distribution of irradiation-induced defect density,

$$
N_{\text {def }}(x)=\left\{\begin{array}{cc}
\left(\frac{x}{L_{\mathrm{d}}}\right)^{n} N_{\mathrm{def}}^{0}, & x \leq L_{\mathrm{d}} \\
0, & x>L_{\mathrm{d}}
\end{array},\right.
$$

where $L_{\mathrm{d}}$ defines the irradiation depth, $N_{\text {def }}^{0}$ is the peak value of the irradiation-induced defect density that occurs at the maximum irradiation depth, and $n \geq 0$ is a parameter to describe the profile of defect distribution. The assumption that the defect density is zero at the sample surface is a simplification made here and is based on the following reasons: (1) in many cases of ion beam irradiation, the irradiation dose near the free surface is rather small compared to the deeper radiation damage [22,37], and therefore is neglected here. (2) Irradiation-induced defects can be absorbed at the free surface in the first few $\mathrm{nm}[43,44]$. However, we do note that our model can be easily modified to account for the non-zero surface defect density. The special case of $n=0$ describes a uniform defect distribution within the irradiated region, $x \leq L_{\mathrm{d}}$. For $n=1$, the irradiation-induced defect density increases linearly from zero on the surface to its maximum value $N_{\text {def }}^{0}$ at $x=L_{\mathrm{d}}$. 


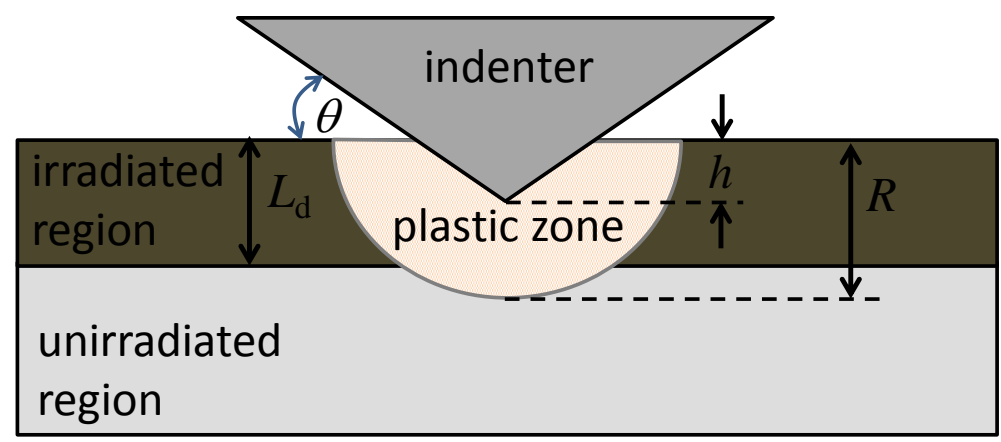

Figure 1. Schematic of the indentation test. The indentation-induced plastic zone is assumed to be a hemisphere.

To compute $\bar{N}_{\text {def }}$ from the $N_{\text {def }}(x)$ above, we need to know the volume of the plastic zone induced by the indenter. As the indenter forces into the material, severe plastic deformation occurs within the region near the indenter tip. The shape and size of the indentation-induced plastic zone may depend on the indenter shape, material properties of tested samples and the loading conditions $[19,45,46]$. For the purpose of developing an explicit analytical model, we assume in this section that the plastic zone is a hemisphere as shown in Fig. 1. This assumption is validated by our numerical simulation results using the finite element method, see the Appendix, which shows that the plastic zone in ion irradiated stainless steel is indeed nearly a hemisphere for the conical-shaped indenter. Further, we note that when the plastic zone has extended beyond the irradiated layer, the radius of the plastic zone inside the irradiated layer may be smaller than that outside the irradiated layer. This is mainly due to the impediment of moving dislocations by irradiation-induced defects in the irradiated layer. However, previous studies [46] and our simulations in the Appendix have indicated that such difference is rather small. Thus it is neglected in this work.

For convenience, the radius of the hemispherical plastic zone is written as $R=M \cdot h$, where $M$ is a dimensionless coefficient and $h$ is the indentation depth. According to the finite element simulations as shown in the Appendix, $M \approx 7.5$ is almost a constant (independent of $h$ ) for conical indenters. The literature value of $M$ ranges from 5 to 10 for Berkovich indenters [7, 28, $47,48]$.

As the indentation depth $h$ increases gradually, so does the size of the plastic zone under the indenter tip. When the plastic zone is still fully contained inside the irradiated zone, i.e., $R \leq L_{\mathrm{d}}$, 
or when $h \leq h_{\mathrm{c}}^{\text {sep }}=L_{\mathrm{d}} / M$, the average defect density in the plastic zone is given by

$$
\bar{N}_{\text {def }}(h)=\frac{\int_{0}^{R} \pi\left(R^{2}-x^{2}\right) N_{\mathrm{def}}^{0}\left(\frac{x}{L_{\mathrm{d}}}\right)^{n} \mathrm{~d} x}{\frac{2}{3} \pi R^{3}}=\frac{3 N_{\mathrm{def}}^{0}(M h)^{n}}{(n+1)(n+3) L_{\mathrm{d}}^{n}} \quad \text { for } h \leq h_{\mathrm{c}}^{\mathrm{sep}},
$$

where $h_{\mathrm{c}}^{\text {sep }}$ is the indentation depth at which the plastic region reaches the maximum depth of the irradiated zone, $L_{\mathrm{d}}$. The average defect density in the plastic zone is calculated by integrating the defect density in the plastic zone, then dividing the integral by the volume of the plastic zone. Here, we have explicitly indicated that $\bar{N}_{\text {def }}$ is a function of the indentation depth $h$.

When the plastic zone is beyond the irradiated region, i.e., when $h>h_{\mathrm{c}}^{\mathrm{sep}}$, the average defect density over the plastic zone is given by

$$
\bar{N}_{\text {def }}(h)=\frac{\int_{0}^{L_{\mathrm{d}}} \pi\left(R^{2}-x^{2}\right) N_{\mathrm{def}}^{0}\left(\frac{x}{L_{\mathrm{d}}}\right)^{n} \mathrm{~d} x}{\frac{2}{3} \pi R^{3}}=\frac{3 N_{\mathrm{def}}^{0} L_{\mathrm{d}}}{2(M h)^{3}}\left[\frac{(M h)^{2}}{n+1}-\frac{L_{\mathrm{d}}^{2}}{n+3}\right], \quad \text { for } h>h_{\mathrm{c}}^{\text {sep }} .
$$

Next, let us consider the average dislocation density $\bar{\rho}_{\text {dis }}$. Within the plastic zone, $\bar{\rho}_{\text {dis }}$ will consist of the spatial averaged geometrically necessary dislocations $\bar{\rho}_{\mathrm{G}}$ and the statistically stored dislocations $\bar{\rho}_{\mathrm{S}}$, i.e., $\bar{\rho}_{\mathrm{dis}}=\bar{\rho}_{\mathrm{G}}+\bar{\rho}_{\mathrm{S}}$. According to the work of Nix and Gao (1998), the total length of geometrically necessary dislocations (i.e., the total length of injected dislocation loops) in the plastic region can be expressed as $\lambda=\pi h^{2} /(b \tan \theta)$, therefore, $\bar{\rho}_{\mathrm{G}}$ yields

$$
\bar{\rho}_{\mathrm{G}}(h)=\frac{\lambda}{V}=\frac{\frac{\pi h^{2}}{b \tan \theta}}{\frac{2}{3} \pi R^{3}}=\frac{3}{2 b M^{3} \tan \theta} \frac{1}{h},
$$

and for convenience, $\bar{\rho}_{\mathrm{S}}$ can be expressed as

$$
\bar{\rho}_{\mathrm{S}}=\frac{3}{2 b \tan \theta} \frac{1}{h^{*}},
$$

where $h^{*}=40.5 b \alpha^{2} \mu^{2} /\left(H_{0}^{2} \tan \theta\right)$ is the characteristic length depending on the statistically stored dislocation density through $H_{0}$, which is the hardness arising from the statistically stored dislocations alone [39]. Finally, one may write 


$$
\bar{\rho}_{\mathrm{dis}}(h)=\bar{\rho}_{\mathrm{G}}+\bar{\rho}_{\mathrm{S}}=\frac{3}{2 b \tan \theta} \frac{1}{h^{*}}\left(1+\frac{\bar{h}^{*}}{h}\right),
$$

where $\bar{h}^{*}=h^{*} / M^{3}$ and $H_{0}=3 \sqrt{3} \mu b \alpha \sqrt{\bar{\rho}_{\mathrm{S}}}$.

Substitution of Eqs. (8), (9) and (12) into Eq. (5) yields the depth dependent hardness of ion irradiated materials,

$$
H_{\text {irr }}= \begin{cases}H_{0} \sqrt{1+\frac{\bar{h}^{*}}{h}+\frac{A^{2} \bar{h}^{*} h^{n}}{(n+1)(n+3)\left(h_{\mathrm{c}}^{\text {sep }}\right)^{n+1}}} & \text { for } h \leq h_{\mathrm{c}}^{\text {sep }} \\ H_{0} \sqrt{1+\frac{\bar{h}^{*}}{h}+\frac{A^{2} \bar{h}^{*}}{2 h}\left[\frac{1}{n+1}-\frac{\left(h_{\mathrm{c}}^{\text {sep }}\right)^{2}}{(n+3) h^{2}}\right]} & \text { for } h>h_{\mathrm{c}}^{\text {sep }}\end{cases}
$$

where $A=\frac{\beta}{\alpha} M \sqrt{2 b \tan \theta L_{\mathrm{d}} N_{\mathrm{def}}^{0} d_{\mathrm{def}}}$ is a coefficient related to the ratio of the hardening coefficients $\alpha$ and $\beta$, the indenter geometry $\theta$, the ratio coefficient $M$, the defect size $d_{\text {def }}$ and the state of irradiation damage represented by $N_{\text {def }}^{0}$ and $L_{\mathrm{d}}$.

A special case of the model developed above is $n=0$ and $L_{\mathrm{d}} \rightarrow \infty$, which corresponds to the case of uniform irradiation damage throughout the thickness of the irradiated sample. In this case, Eq. (13) reduces to

$$
H_{\mathrm{irr}}=H_{0} \sqrt{1+\frac{2 \beta^{2}}{3 \alpha^{2}} \tan \theta N_{\mathrm{def}}^{0} d_{\mathrm{def}} b h^{*}+\frac{\bar{h}^{*}}{h}}=H_{\mathrm{irr}}^{0} \sqrt{1+\frac{h_{\mathrm{irr}}^{0}}{h}},
$$

where

$$
H_{\text {irr }}^{0}=3 \sqrt{3} \mu b \sqrt{\frac{3 \alpha^{2}+2 b \tan \theta N_{\text {def }}^{0} d_{\text {def }} h^{*} \beta^{2}}{2 b \tan \theta h^{*}}} \text { and } h_{\mathrm{irr}}^{0}=\frac{3 \alpha^{2} \bar{h}^{*}}{3 \alpha^{2}+2 b \tan \theta N_{\text {def }}^{0} d_{\text {def }} h^{*} \beta^{2}} .
$$

We note that the depth-dependent hardness in the case of uniform irradiation damage is similar in form to that of the Nix-Gao model, which has been used to fit the experimental data of ion irradiated materials when $h \leq h_{\mathrm{c}}^{\text {sep }}[7,8,19-27]$. However, knowing that Eq. (14) is valid only for uniform defect distribution, and ion irradiation often produces non-uniform defect distribution, we can now appreciate that Eq. (14) is not appropriate for ion irradiated samples.

It is also easy to see that the model developed here can be easily reduced to the Nix-Gao model [33] for unirradiated materials, where $\rho_{\text {def }}^{0}=0$. Thus, Eq. (14) reduces to 


$$
H_{\text {unirr }}=H_{0} \sqrt{1+\frac{\bar{h}^{*}}{h}}=H_{0} \sqrt{1+\frac{1}{M^{3}} \frac{h^{*}}{h}},
$$

here Eq. (16) is identical to the expression given in [33], if $M$ is assumed to be $M=1 / \tan \theta$ as did in [33].

\section{Parameterization of the Model}

The model presented in Eq. (13) contains 11 parameters, which can be classified into groups. For example, $N_{\text {def }}^{0}, L_{d}$ and $n$ describe the state of irradiation damage; $\mu$ and $b$ indicate the intrinsic material properties of the unirradiated material; $\bar{\rho}_{\mathrm{S}}, \alpha$ and $\beta$ describe the density of statistically stored dislocations, and the strength of dislocations and defects, respectively; And, $\theta$ defines the geometry of the indenter. All of these are intrinsic to the material being indented. In addition, there is the parameter $M$ that describes the size of the plastic zone which is related to the loading. Some of these parameters, such as $\theta, \mu$ and $b$ are generally known prior to the indentation test. Others like $N_{\text {def }}^{0}, L_{d}, n, \bar{\rho}_{\mathrm{S}}, \alpha$ and $\beta$ are usually not known a priori.

Although there are 11 physical parameters, only five combinations of them appear explicitly in Eq. (13). Among them, $H_{0}$ and $\bar{h}^{*}$ are independent of the irradiation, while $n, A$ and $h_{\mathrm{c}}^{\text {sep }}$ are related to the irradiated materials. In this section, we will describe a method to experimentally obtain these five parameters by fitting the model to the experimental data.

First, since $H_{0}$ and $\bar{h}^{*}$ are independent of irradiation, one can conduct an indentation test on the unirradiated material to obtain its hardness $\tilde{H}_{\text {unirr }}$ as a function of the indentation depth $h$, i.e., the $\tilde{H}_{\text {unirr }}$ vs. $h$ curve, where and in the rest of this paper, a tilt $\sim$ over a quantity mean that that quantity is measured experimentally. It then follows from Eq. (16) that the $\left(\tilde{H}_{\text {unirr }}\right)^{2}$ vs. $1 / h$

curve is a straight line. Its intercept with the vertical axis is $H_{0}^{2}$ and its slope is $H_{0}^{2} \bar{h}^{*}$ [33]. This yields the values of $H_{0}$ and $\bar{h}^{*}$.

Next, we consider the remaining three parameters $h_{\mathrm{c}}^{\text {sep }}, n$, and $A$. To this end, one needs to conduct an indentation test on an irradiated material to obtain its harness $\tilde{H}_{\text {irr }}$ vs. $h$ curve. Since $H_{0}$ and $\bar{h}^{*}$ are known by now, the experimentally obtained $\tilde{H}_{\text {irr }}$ vs. $h$ curve can be transformed 
into the $\tilde{f}(h) \equiv\left(\frac{\tilde{H}_{\text {irr }}}{H_{0}}\right)^{2}-\frac{\bar{h}^{*}}{h}-1$ vs. $h$ curve, which according to Eq. (13) should be described by

$$
f(h) \equiv\left(\frac{H_{\mathrm{irr}}}{H_{0}}\right)^{2}-\frac{\bar{h}^{*}}{h}-1=\left\{\begin{array}{ll}
P h^{n} & \text { for } h \leq h_{\mathrm{c}}^{\text {sep }} \\
Z \frac{1}{h}-Q \frac{1}{h^{3}} & \text { for } h>h_{\mathrm{c}}^{\text {sep }}
\end{array},\right.
$$

where

$$
P=\frac{A^{2} \bar{h}^{*}}{(n+1)(n+3)\left(h_{\mathrm{c}}^{\mathrm{sep}}\right)^{n+1}}, Z=\frac{P}{2}(n+3)\left(h_{\mathrm{c}}^{\mathrm{sep}}\right)^{n+1} \text { and } Q=\frac{P}{2}(n+1)\left(h_{\mathrm{c}}^{\mathrm{sep}}\right)^{n+3} .
$$

Therefore, the goal here is to obtain the parameters $P, Z, Q$ and $n$ by fitting the $f(h)$ defined in Eq. (17) with the experimentally measured $\tilde{f}(h)$ vs. $h$ curve.

To begin, we note that $h_{\mathrm{c}}^{\text {sep }}$ is not known a prior. Therefore, it is somewhat ambiguous in identifying the experimental data for the region $h \geq h_{\mathrm{c}}^{\text {sep }}$. To overcome this difficulty, it is informative to study the behavior of Eq. (17). Note that in the region $h>h_{\mathrm{c}}^{\text {sep }}$, $f(h)=Z / h-Q / h^{3}$ first increases with increasing $h$ until it reaches a threshold depth $h_{\mathrm{c}}^{\max }$ at which $f(h)=Z / h-Q / h^{3}$ achieves its maximum value. For $h>h_{\mathrm{c}}^{\max }, f(h)=Z / h-Q / h^{3}$ decreases monotonously with increasing $h$. This non-monotonic behavior can be explained as follows. With increasing $h$ when $h \leq h_{\mathrm{c}}^{\text {sep }}$, the plastic zone expands deeper into the material that has a higher defect density, see Eq. (8). So the average defect density over the plastic zone will increase. Thus more hardening occurs due to the increased dislocation barriers. This situation continues even after the bottom portion of the plastic zone has extruded into the irradiationdamage-free zone, $x>L_{\mathrm{d}}$ or $h>h_{\mathrm{c}}^{\mathrm{sep}}$, because the expansion of the hemispherical plastic zone continues to include more volume of the material that contains a higher defect density so the average defect density over the plastic zone still increases with increasing $h$. The situation will change, however, after $h=h_{\mathrm{c}}^{\max }$ when the portion of the plastic zone below the irradiated zone is sufficiently large that the average defect density over the plastic zone begins to decrease with further increasing $h$. This threshold value $h_{\mathrm{c}}^{\max }$ can be obtained by calculating the derivative of $f(h)=Z / h-Q / h^{3}$ with respect to $h$, and setting it to zero, i.e., $d\left(Z / h-Q / h^{3}\right) / d h=0$, which yields 


$$
h_{\mathrm{c}}^{\max }=\sqrt{\frac{3(n+1)}{n+3}} h_{\mathrm{c}}^{\mathrm{sep}} .
$$

From the experimentally obtained $\tilde{f}(h)$ vs. $h$ curve, the value of $h_{\mathrm{c}}^{\max }$ can be easily extracted, because it is at $h=h_{\mathrm{c}}^{\max }$ that $\tilde{f}(h)$ achieves its maximum value. With $h_{\mathrm{c}}^{\max }$ being known from $\tilde{f}(h)$, Eq. (19) provides an equation for the unknown parameters $n$ and $h_{\mathrm{c}}^{\text {sep }}$. Furthermore, since $n \geq 0$, Eq. (19) also indicates that $h_{\mathrm{c}}^{\max } \geq h_{\mathrm{c}}^{\text {sep }}$. Thus, for $h>h_{\mathrm{c}}^{\max }$, it is assured that the correct expression to use is $f(h)=Z / h-Q / h^{3}$. In other words, by fitting $f(h)=Z / h-Q / h^{3}$ with $\tilde{f}(h)$ in the region $h>h_{\mathrm{c}}^{\max }$, one can obtain the values for $Z$ and $Q$.

Next, since $Z$ and $Q$ are known now, we can plot both the experimental data $\tilde{f}(h)$ and the fitted function $f(h)=Z / h-Q / h^{3}$ on a log-log scale for the entire range of $h$. The $\log [\tilde{f}(h)]$ vs. $\log [h]$ curve should be a straight line in the region $h \leq h_{\mathrm{c}}^{\text {sep }}$. Its slope gives $n$ and its intercept with the vertical axis gives $P$. Furthermore, the intersection between this straight line and the $\log$ - $\log$ plot of $f(h)=Z / h-Q / h^{3}$ gives $h_{\mathrm{c}}^{\text {sep }}$.

Finally, using the values of $n, h_{\mathrm{c}}^{\mathrm{sep}}, \bar{h}^{*}$ and $P$ obtained so far, one can compute the value of $A$ from Eq. (18). This completes the parameterization of the model. In summary, the parameterization procedure to determine the five physical parameters $\left(H_{0}, \bar{h}^{*}, h_{\mathrm{c}}^{\mathrm{sep}}, n\right.$ and $\left.A\right)$ in the model described by Eq. (13) consists of the following steps.

(i) Conduct an indentation test on the unirradiated material to obtain the $\tilde{H}_{\text {unirr }}$ vs. $h$ curve.

(ii) Conduct an indentation test on an irradiated material to obtain the $\tilde{H}_{\text {irr }}$ vs. $h$ curve.

(iii) Replot the experimental data from the unirradiated material into the $\left(\tilde{H}_{\text {unirr }}\right)^{2}$ vs. $1 / h$ curve, which should be represented by a straight line. Its intercept is $H_{0}^{2}$ and its slope is $H_{0}{ }^{2} \bar{h}^{*}$. This yields the parameters $H_{0}$ and $\bar{h}^{*}$.

(iv) Replot the experimental data from the irradiated material into the $\tilde{f}(h) \equiv\left(\frac{\tilde{H}_{\text {irr }}}{H_{0}}\right)^{2}-\frac{\bar{h}^{*}}{h}-1$ vs. $h$ curve. This curve has a maximum value at $h=h_{\mathrm{c}}^{\max } . \mathrm{We}$ 
will record this value of $h_{\mathrm{c}}^{\max }$ for use at the next steps.

(v) Use a nonlinear regression method to fit $f(h)=Z / h-Q / h^{3}$ with $\tilde{f}(h)$ in the region $h>h_{\mathrm{c}}^{\max }$. This will yield the values for $Q$ and $Z$.

(vi) Replot the $\tilde{f}(h)$ vs. $h$ curve and the $f(h)=Z / h-Q / h^{3}$ vs. $h$ curve on a log-log scale. The $\log [\tilde{f}(h)]$ curve should be a straight line in the region $h \leq h_{\mathrm{c}}^{\mathrm{sep}}$. Its slope gives $n$ and its intercept with the vertical axis gives $P$. Furthermore, the intersection between this straight line and the log-log plot of $f(h)=Z / h-Q / h^{3}$ gives $h_{\mathrm{c}}^{\text {sep }}$.

(vii) Compute the parameter $A$ from Eq. (18).

\section{Model Validation using Experimental Data for Stainless Steels}

In this section, we will use existing experimental data to test whether the model developed here can be used to simulate the depth dependent hardness of ion irradiated materials.

We begin with the experimental data presented in [30] for stainless steels. The material used in this study is an austenite stainless steel. The specimens were irradiated with $240 \mathrm{keV}$ protons at room temperature. More details about the material composition and the irradiation conditions can be found in [30].

Following the fitting procedure described in the previous section, we obtained the model parameters corresponding to the experimental data. These fitted parameters are listed in Table 1.

Table 1. Fitted model parameters for the proton irradiated stainless steel [30].

\begin{tabular}{c|c|c}
\hline Material & \multicolumn{2}{|c}{ Stainless steels [30] } \\
\hline Irradiation dose (dpa) & 2 & 7 \\
\hline$H_{0}(\mathrm{GPa})$ & \multicolumn{2}{|c}{2.73} \\
\hline$h^{*}(\mathrm{~nm})$ & 591 & 805 \\
\hline$Z(\mathrm{~nm})$ & $6.15 \times 10^{6}$ & $9.42 \times 10^{6}$ \\
\hline$Q\left(\mathrm{~nm}^{3}\right)$ & 1.264 & 1.278 \\
\hline$n$ & & 290 \\
\hline
\end{tabular}




\begin{tabular}{c|c|c}
\hline$P\left(\mathrm{~nm}^{-\mathrm{n}}\right)$ & $3.8 \times 10^{-3}$ & $5.3 \times 10^{-3}$ \\
\hline$h_{\mathrm{c}}^{\text {sep }}(\mathrm{nm})$ & 140 & 141 \\
\hline$A$ & 2.948 & 3.65 \\
\hline
\end{tabular}

Using these fitted parameters in Eq. (17), we plotted the $f(h)$ against the experimental data $\tilde{f}(h)$. The results are shown in Fig. 2(a). Similarly, we plotted the hardness as a function of indentation depth in Fig. 2(b), and compared the results from our parameterized model with that of the experiments. In both figures, the symbols are the experimental data and the solid lines are from our parameterized model.
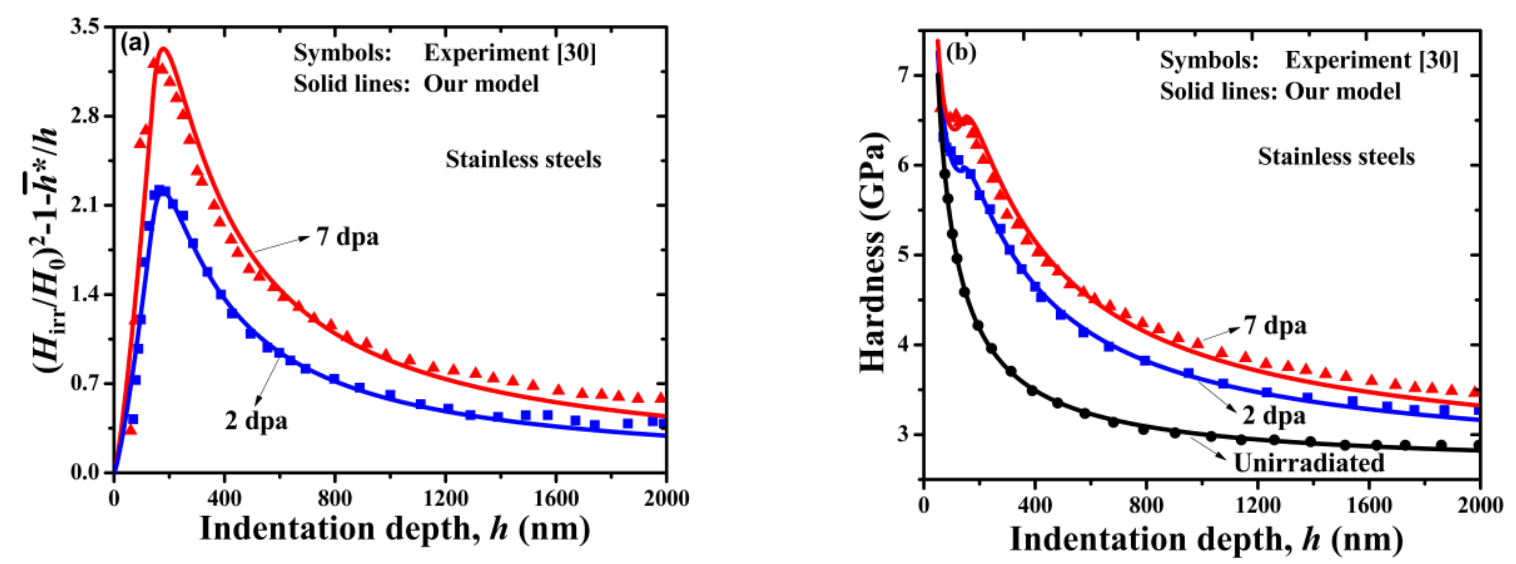

Figure 2. Comparison between model prediction and experimental data from [30].

It is seen from these figures that our parameterized model reproduces the experimental results with very good accuracy for this particular stainless steels under different irradiation conditions. In addition, our model also provides more insight into the material behavior. For example, it is seen from Fig. 2(b) that the indentation size effects is obvious when the indentation depth is within a few hundreds of nanometers, and it exists in both irradiated and unirradiated materials. Further, the indentation depth can be divided into three regions by two critical depths, i.e. $h_{\mathrm{c}}^{\text {sep }}$ and $h_{\mathrm{c}}^{\max }$. In the region $h \leq h_{\mathrm{c}}^{\text {sep }}$, the irradiation hardening behavior is only affected by the inhomogeneously distributed defects in the irradiated region. In the region $h_{\mathrm{c}}^{\mathrm{sep}}<h \leq h_{\mathrm{c}}^{\max }$, the plastic zone is beyond the irradiated region, and the unirradiated substrate starts to affect the hardening behavior, whereas the average defect density still increases with $h$ until $h=h_{\mathrm{c}}^{\max }$ when 
the unirradiated substrate begins to weaken the hardening behavior.

To investigate the robustness of our model, we consider another set of experimental data [23]. The material used in this study is again a stainless steel called China A508-3 steels. The samples were irradiated by $240 \mathrm{keV} \mathrm{H}^{+}$ions in a vacuum chamber at $0.11 \mathrm{dpa}$ and at $0.5 \mathrm{dpa}$, respectively. Details about the material and the irradiation conditions are given in [23].

Following the procedures developed in Sec. 3, our hardness model with irradiation effect was parameterized with the experimental data in [23]. The fitted parameters are listed in Table 2. The comparison between the experimental data and the results from our parameterized model is showing in Fig. 3. Again, we see that the results from our parameterized model follow the experimental data closely.

Table 2. Fitted model parameters for the ion irradiated China A508-3 steels [23].

\begin{tabular}{c|c|c}
\hline Material & \multicolumn{2}{|c}{ China A508-3 steels [23] } \\
\hline$H_{0}(\mathrm{GPa})$ & 0.11 & 0.5 \\
\hline$h^{*}(\mathrm{~nm})$ & \multicolumn{2}{|c}{2.35} \\
\hline$Z(\mathrm{~nm})$ & 507 & 226 \\
\hline$Q\left(\mathrm{~nm}{ }^{3}\right)$ & $1.59 \times 10^{7}$ & 725 \\
\hline$n$ & 0.61 & 0.41 \\
\hline$P\left(\mathrm{~nm}^{-\mathrm{n}}\right)$ & 0.035 & 0.201 \\
\hline$h_{\mathrm{c}}^{\mathrm{sep}}(\mathrm{nm})$ & 265 & 225 \\
\hline$A$ & 2.678 & 2.977 \\
\hline
\end{tabular}



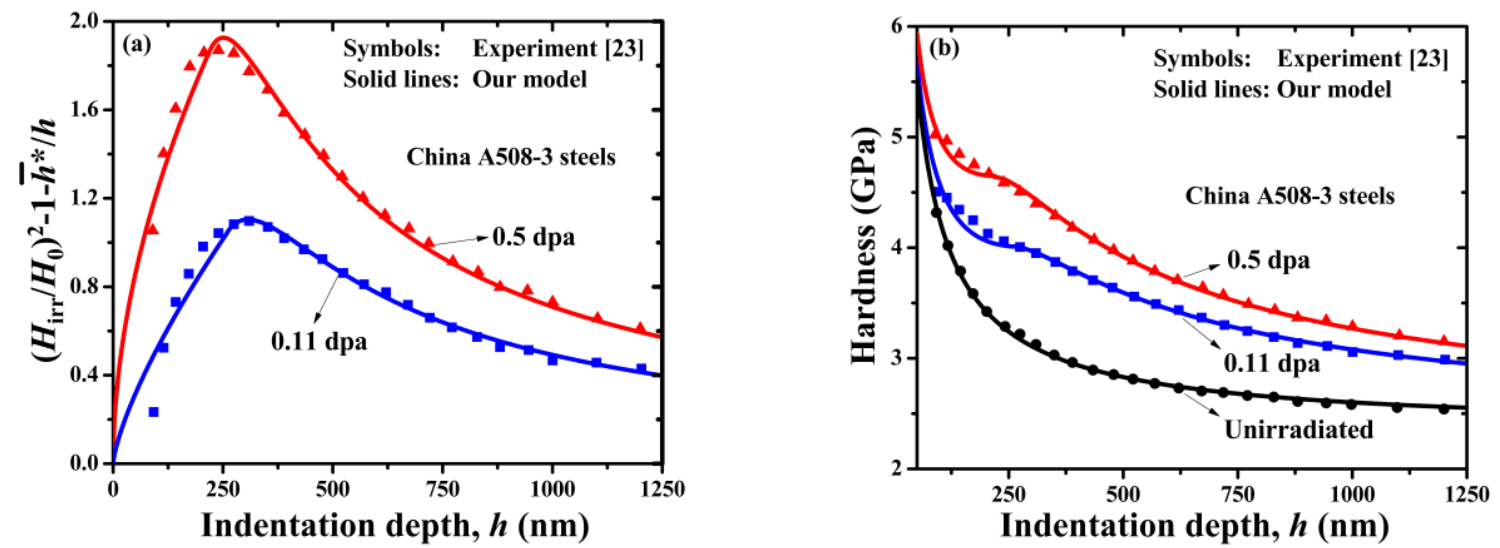

Figure 3. Comparison between model prediction and experimental data from [23].

Finally, we studied the 16MND5 steels irradiated by Fe-ions at $1.37 \mathrm{dpa}$ and protons at 0.5 dpa [22]. After obtaining the values of $H_{0}$ and $\bar{h}^{*}$ from the experimental $\tilde{H}_{\text {unirr }}$ vs. $h$ for the unirradiated material, we plotted $\tilde{f}(h) \equiv\left(\frac{\tilde{H}_{\text {irr }}}{H_{0}}\right)^{2}-\frac{\bar{h}^{*}}{h}-1$ vs. $h$ curve in Fig. 4(a). It turns out that we were only able to fit $f(h)=Z / h-Q / h^{3}$ with the experimental data for $h>h_{\mathrm{c}}^{\max }$, which yields the values of $Z$ and $Q$. We were not able to fit $f(h)=P h^{n}$ to the experimental curve when $h<h_{\mathrm{c}}^{\text {sep }}$. We speculate that the experiment data in such shallow indentation depth may have been affected by the surface conditions, such as surface roughness, oxidation after irradiation, and/or surface hardening due to sample polishing [46]. Thus, we were not able to obtain the values of $P$, $n$ and $h_{\mathrm{c}}^{\text {sep }}$. The fitted values of $Z, Q, H_{0}$ and $\bar{h}^{*}$ are listed in Table 3 . The model prediction of the hardness is plotted in Fig. 4(b) against the experimental data. Since $h_{\mathrm{c}}^{\text {sep }}, P$ and $n$ are unknown, the model prediction is shown in Fig. 4(b) only for $h>h_{\mathrm{c}}^{\max }$.

Table 3. Parameters for the hardness model with irradiation effect based on the experimental data of ion irradiated 16MND5 steels [22].

\begin{tabular}{c|c|c}
\hline Material & \multicolumn{2}{|c}{ 16MND5 steels [22] } \\
\hline Irradiation dose (dpa) & 1.37 (Fe-ions) & 0.5 (proton) \\
\hline
\end{tabular}




\begin{tabular}{c|c|c}
\hline$H_{0}(\mathrm{GPa})$ & \multicolumn{2}{|c}{2.33} \\
\hline $\bar{h}^{*}(\mathrm{~nm})$ & 313 & 654 \\
\hline$Z(\mathrm{~nm})$ & $2.62 \times 10^{6}$ & $5.66 \times 10^{6}$ \\
\hline$Q\left(\mathrm{~nm}^{3}\right)$ & - & - \\
\hline$n$ & - & - \\
\hline$P\left(\mathrm{~nm}^{-\mathrm{n}}\right)$ & - & - \\
\hline$h_{\mathrm{c}}^{\text {sep }}(\mathrm{nm})$ & & \\
\hline
\end{tabular}
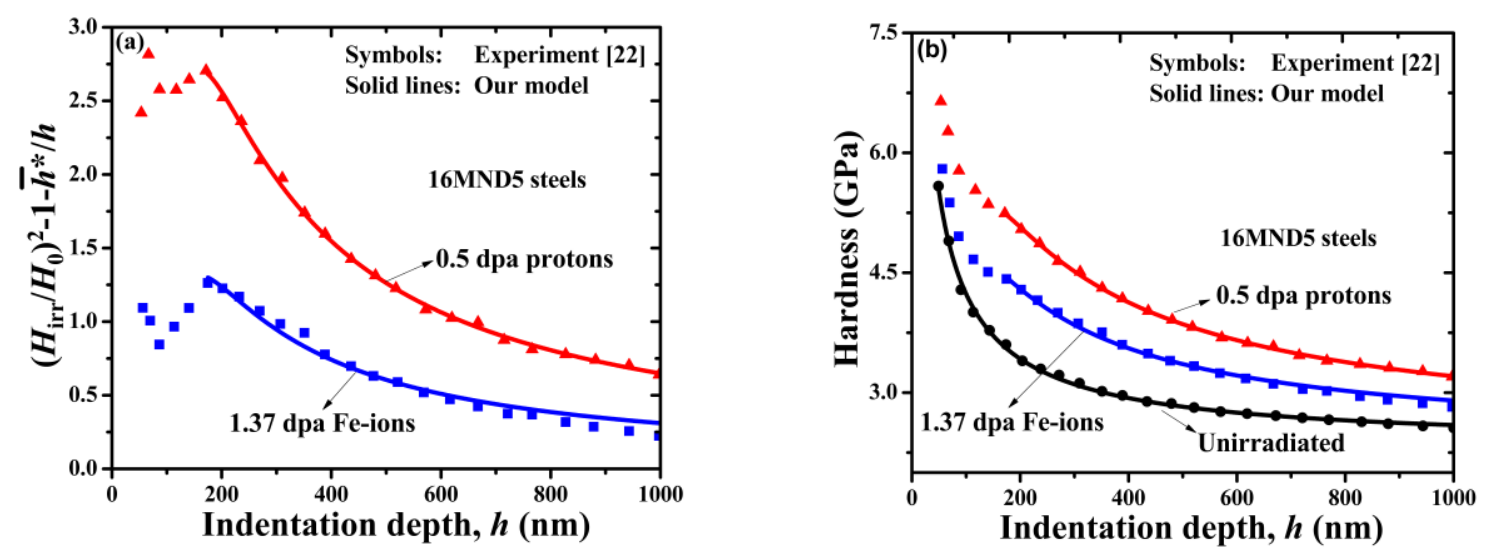

Figure 4. Comparison between model prediction and experimental data from [22].

\section{Conclusions}

A mechanistic model was developed in this paper for modeling the depth-dependent hardness in ion irradiated metallic materials. The model is capable of capturing the indentation size effect, the ion irradiation induced damage gradient effect, and the effect of unirradiated region acting as a soft substrate. A procedure was developed and described in detail in the paper to parametrize the model based on experimentally obtained hardness vs. indentation depth curves. Very good agreement was observed between our model predictions and existing experimental data on several different stainless steels subjected to various ion irradiation conditions.

More importantly, our model reveals several interesting features that play key roles in the deformation process during the indentation of ion irradiated materials. Fundamentally, there are two hardening mechanisms. One is the well-known indentation size effect arising from the 
creation of geometrically necessary dislocations as the indenter pierces into the materials. The other is the irradiation hardening due to the presence of irradiation-induced defects. As a function of indentation depth, the hardening due to indentation size effect is described by $\bar{h}^{*} / h$, and remains so throughout the indentation depth. Clearly, such indentation size effect is important only for small $h$.

On the other hand, the irradiation hardening has a rather complicated depth-dependence. In the shallow indentation regime when the plastic zone is entirely contained within the irradiated layer, i.e., $h<h_{\mathrm{c}}^{\text {sep }}$, irradiation hardening follows a power law form $P h^{n}$, where $n>0$. This is distinct from the hardening behavior $\bar{h}^{*} / h$ induced by the geometrically necessary dislocations. Clearly, $h^{n} \square 1 / h$ for small $h$. In other words, indentation size effect is dominant in the shallow indentation regime.

When the indentation depth exceeds $h>h_{\mathrm{c}}^{\text {sep }}$, i.e., when the plastic zone extends beyond the irradiated layer, and extruded into the unirradiated region of the material, the hardening behavior due to irradiation-induced defects changes to $Z / h-Q / h^{3}$. This indicates that irradiation hardening continues to increase with the indentation depth $h$ until it reaches a threshold depth $h_{\mathrm{c}}^{\max }$ at which irradiation hardening achieves its maximum value. For $h>h_{\mathrm{c}}^{\max }$, irradiation hardening starts to decrease monotonously with indentation depth. This non-monotonic behavior can be potentially exploited to characterize the thickness of the irradiated layer and the distribution of irradiation-induced defects.

As part of the model development, we also conducted numerical analyses based on the finite element method to simulate the deformation process during the indentation of ion irradiated materials. Results of our numerical simulations show that the plastic zone can be well approximated by a hemisphere when the indenter has a conical tip. The size of the plastic zone is approximately 7.5 times of the indentation depth.

\section{Acknowledgements}

This research was supported in part by the US Department of Energy through DE-NE0000678. HD acknowledges the support from the National Natural Science Foundation of China (NSFC) under Grants 11225208, 11521202 and 11632001, and from the key subject "Computational 
Solid Mechanics" of China Academy of Engineering Physics.

\section{Appendix}

In this section, we present the numerical simulation results of indentation tests on ion irradiated materials. The objectives are (1) to investigate the shape of the plastic zone, and (2) to study the relationship between the size of the plastic zone and the indentation depth.

The numerical simulations were conducted using the finite element method. The commercial software ABAQUS was used for this purpose. Because of the axisymmetric nature of the configuration, quadrilateral axisymmetric elements were used in constructing the finite element model.

In the axisymmetric simulation, a conical-shaped indenter with a $70.3^{0}$ semi-angle was used. As pointed out in [49], results from such conical model can also be used to simulate a Berkovich indenter with a similar area-to-depth ratio. The sample to be indented is a cylinder shaped block. Its size is large enough so that the boundaries have no effects on the indented region.

The material considered here is a stainless steel. To simulate ion irradiation damage, the top surface layer of the material is assumed to have a distribution of irradiation-induced defects. The

defect distribution is described by Eq. (7) with $n=1, N_{\text {def }}^{0}=2 \times 10^{23} \mathrm{~m}^{-3}, d_{\text {def }}=1.6 \mathrm{~nm}$ and $L_{\mathrm{d}}=1,050 \mathrm{~nm}$.

An elastic-plastic constitutive law was used in the finite element calculations. The uniaxial stress-strain relationship was derived based on the crystal plasticity model and the self-consistent method developed in $[50,51]$. Material parameters used in this model were calibrated with the experimental data of neutron irradiated stainless steels [52]. Shown in Fig. A.1 is a comparison between the experimental stress-strain curves [52] and the corresponding model results with various levels of defect density. 


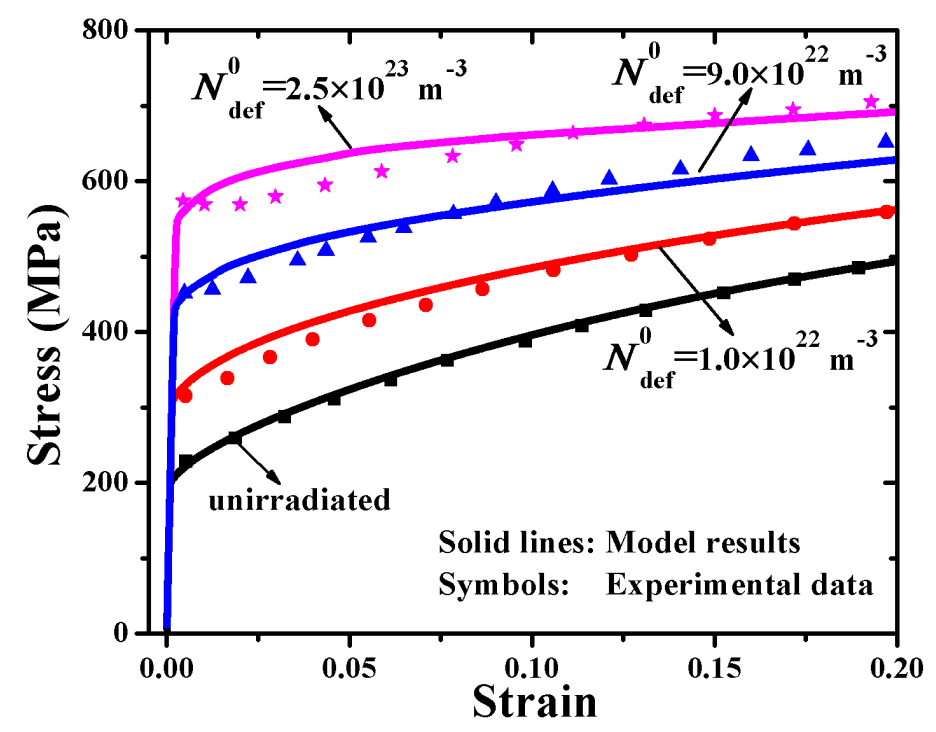

Figure A.1. Uniaxial stress-strain curves for the stainless steel considered here with various densities of irradiation-induced defects. Symbols are the experimental data from [52] and solid lines are from the crystal plasticity self-consistent method [50,51].

Because we are interested in the material behavior in shallow indentation depth (nanoindentation), surface size effect may play a role. To account for the indentation size effect, the mechanism-based strain gradient plasticity [33] with a material length, $l=5 \mu \mathrm{m}$ was used in conjunction with the crystal plasticity model described in the previous paragraph. This was accomplished by implementing in the ABAQUS with a user subroutine.

Shown in Fig. A.2 is a contour plot of the equivalent plastic strain at the indentation depth of $2 \mu \mathrm{m}$. These contours represent the shape of the plastic zone. It is seen that the plastic zone can be reasonably approximated by a hemisphere.

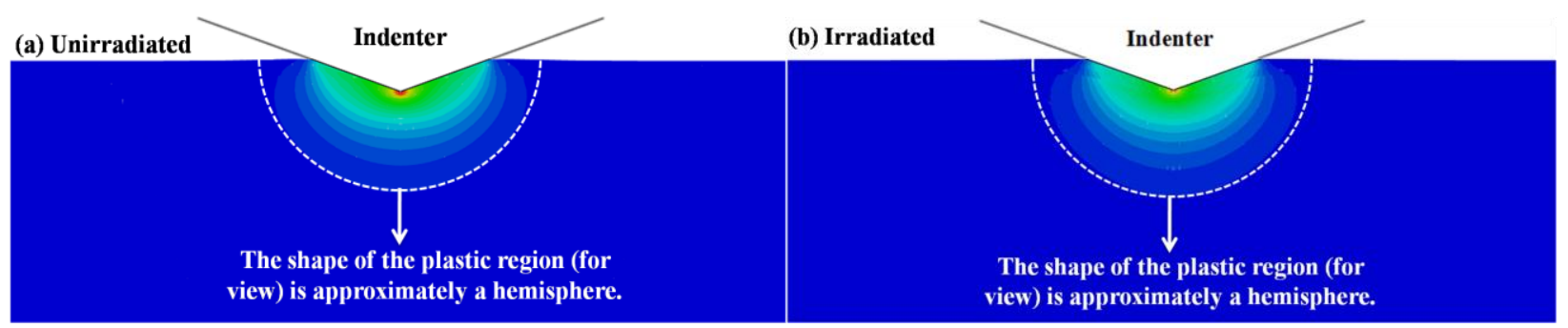

Figure A.2. Contour plots of the equivalent plastic strain in (a) an unirradiated sample and (b) an 
irradiated sample at the indentation depth of $2 \mu \mathrm{m}$. The semi-circle is added to visually illustrate that the plastic zone, represented here by the contours of the equivalent plastic strain, is approximately a hemisphere.

The size of the plastic zone $R$ was also computed as a function of the indentation depth $h$. Show in Fig. A. 3 is the ratio $M=R / h$ as a function of $h$. It is seen that for deep enough ( $h>400$ $\mathrm{nm}$ ) indentation depth, the ratio $M$ is almost a constant for both unirradiated and irradiated cases, i.e., the size of the plastic zone is proportional to the indentation depth.

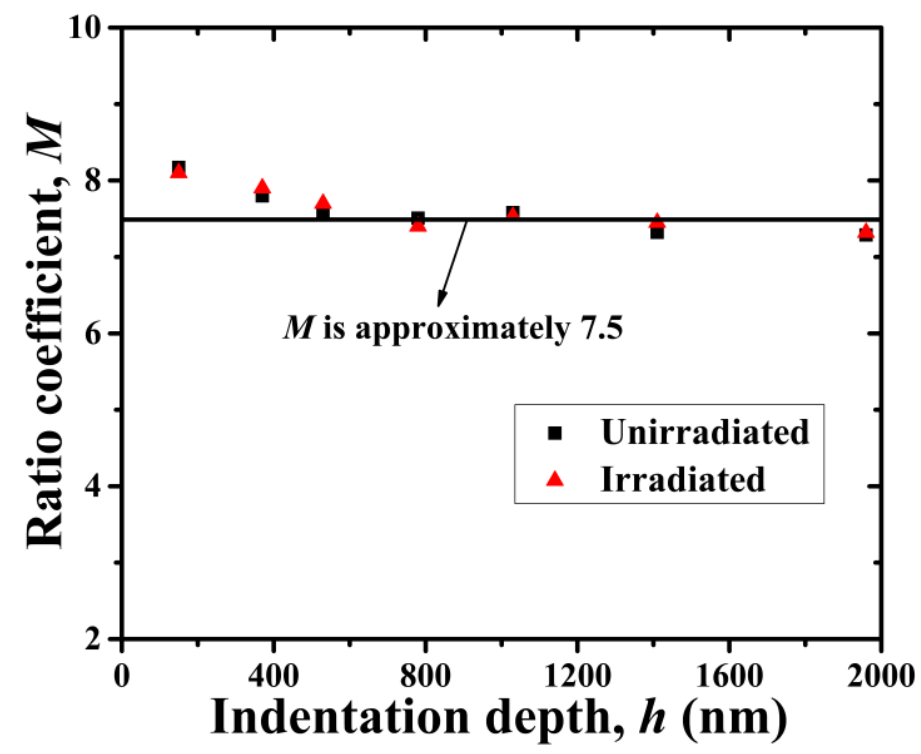

Figure A.3. Coefficient $M$ as a function of the indentation depth for both the irradiated and unirradiated cases. $M$ is almost a constant value of 7.5 when the indentation depth is over $400 \mathrm{~nm}$. 


\section{Reference}

[1] T.S. Byun, K. Farrell, Plastic instability in polycrystalline metals after low temperature irradiation, Acta Materialia 52(6) (2004) 1597-1608.

[2] T.S. Byun, N. Hashimoto, K. Farrell, E.H. Lee, Characteristics of microscopic strain localization in irradiated 316 stainless steels and pure vanadium, Journal of Nuclear Materials 349(3) (2006) 251-264.

[3] T.S. Byun, N. Hashimoto, Strain Hardening During Mechanical Twinning and Dislocation Channeling in Irradiated 316 Stainless Steels, Effects of Radiation on Materials: 23rd International Symposium 1492 (2008) 121-133.

[4] P.J. Maziasz, Overview of microstructural evolution in neutron irradiated austentic stainless steels, Journal of Nuclear Materials 205 (1993) 118-145.

[5] K. Yabuuchi, Y. Kuribayashi, S. Nogami, R. Kasada, A. Hasegawa, Evaluation of irradiation hardening of proton irradiated stainless steels by nanoindentation, Journal of Nuclear Materials 446(1-3) (2014) 142-147.

[6] Z. Jiao, G. Was, T. Miura, K. Fukuya, Aspects of ion irradiations to study localized deformation in austenitic stainless steels, Journal of Nuclear Materials 452(1-3) (2014) 328-334.

[7] H.F. Huang, J.J. Li, D.H. Li, R.D. Liu, G.H. Lei, Q. Huang, L. Yan, TEM, XRD and nanoindentation characterization of Xenon ion irradiation damage in austenitic stainless steels, Journal of Nuclear Materials 454(1-3) (2014) 168-172.

[8] Z.Y. Fu, P.P. Liu, F.R. Wan, Q. Zhan, Helium and hydrogen irradiation induced hardening in CLAM steel, Fusion Engineering and Design 91 (2015) 73-78.

[9] C. Heintze, F. Bergner, S. Akhmadaliev, E. Altstadt, Ion irradiation combined with nanoindentation as a screening test procedure for irradiation hardening, Journal of Nuclear Materials 472 (2016) 196-205.

[10] R. Kasada, S. Konishi, K. Yabuuchi, S. Nogami, M. Ando, D. Hamaguchi, H. Tanigawa, Depth-dependent nanoindentation hardness of reduced-activation ferritic steels after $\mathrm{MeV} F e$-ion irradiation, Fusion Engineering and Design 89(7-8) (2014) 1637-1641.

[11] D. Chen, K. Murakami, K. Dohi, K. Nishida, N. Soneda, Z. Li, L. Liu, N. Sekimura, Depth distribution of Frank loop defects formed in ion-irradiated stainless steel and its dependence on Si addition, Nuclear Instruments \& Methods in Physics Research Section B-Beam Interactions with Materials and Atoms 365 (2015) 503-508.

[12] P. Hosemann, D. Kiener, Y. Wang, S.A. Maloy, Issues to consider using nano indentation on shallow ion beam irradiated materials, Journal of Nuclear Materials 425(1-3) (2012) 136-139. 
[13] W.C. Oliver, G.M. Pharr, Measurement of hardness and elastic modulus by instrumented indentation: Advances in understanding and refinements to methodology, Journal of Materials Research 19(1) (2004) 3-20.

[14] W.C. Oliver, G.M. Pharr, An improved technique for determining hardness and elastic modulus using load and displacement sensing indentation experiments, Journal of Materials Research 7(6) (1992) 1564-1583.

[15] J.T. Busby, M.C. Hash, G.S. Was, The relationship between hardness and yield stress in irradiated austenitic and ferritic steels, Journal of Nuclear Materials 336(2-3) (2005) 267-278.

[16] D. Kiener, A.M. Minor, O. Anderoglu, Y. Wang, S.A. Maloy, P. Hosemann, Application of small-scale testing for investigation of ion-beam-irradiated materials, Journal of Materials Research 27(21) (2012) 2724-2736.

[17] C.D. Hardie, S.G. Roberts, Nanoindentation of model Fe-Cr alloys with self-ion irradiation, Journal of Nuclear Materials 433(1-3) (2013) 174-179.

[18] Z.X. Zhang, D.S. Chen, W.T. Han, A. Kimura, Irradiation hardening in pure tungsten before and after recrystallization, Fusion Engineering and Design 98-99 (2015) 2103-2107.

[19] C.D. Hardie, S.G. Roberts, A.J. Bushby, Understanding the effects of ion irradiation using nanoindentation techniques, Journal of Nuclear Materials 462 (2015) 391-401.

[20] C. Heintze, F. Bergner, M. Hernandez-Mayoral, Ion-irradiation-induced damage in $\mathrm{Fe}-\mathrm{Cr}$ alloys characterized by nanoindentation, Journal of Nuclear Materials 417(1-3) (2011) 980-983.

[21] P. Hosemann, C. Vieh, R.R. Greco, S. Kabra, J.A. Valdez, M.J. Cappiello, S.A. Maloy, Nanoindentation on ion irradiated steels, Journal of Nuclear Materials 389(2) (2009) 239-247.

[22] X. Liu, R. Wang, A. Ren, J. Jiang, C. Xu, P. Huang, W. Qian, Y. Wu, C. Zhang, Evaluation of radiation hardening in ion-irradiated Fe based alloys by nanoindentation, Journal of Nuclear Materials 444(1-3) (2014) 1-6.

[23] X. Liu, R. Wang, J. Jiang, Y. Wu, C. Zhang, A. Ren, C. Xu, W. Qian, Slow positron beam and nanoindentation study of irradiation-related defects in reactor vessel steels, Journal of Nuclear Materials 451(1-3) (2014) 249-254.

[24] Y. Yang, C. Zhang, Y. Meng, J. Liu, J. Gou, Y. Xian, Y. Song, Nanoindentation on V-4Ti alloy irradiated by H and He ions, Journal of Nuclear Materials 459 (2015) 1-4.

[25] M. Roldan, P. Fernandez, J. Rams, D. Jimenez-Rey, E. Materna-Morris, M. Klimenkov, Comparative study of helium effects on EU-ODS EUROFER and EUROFER97 by nanoindentation and TEM, Journal of Nuclear Materials 460 (2015) 226-234. 
[26] Y. Takayama, R. Kasada, Y. Sakamoto, K. Yabuuchi, A. Kimura, M. Ando, D. Hamaguchi, H. Tanigawa, Nanoindentation hardness and its extrapolation to bulk-equivalent hardness of F82H steels after single- and dual-ion beam irradiation, Journal of Nuclear Materials 442(1-3) (2013) S23-S27.

[27] J. Hu, X. Wang, Y. Gao, Z. Zhuang, T. Zhang, Q. Fang, C. Liu, Microstructure and NanoHardness of $10 \mathrm{MeV}$ Cl-Ion Irradiated T91 Steel, Plasma Science \& Technology 17(12) (2015) 1088-1091.

[28] T. Miura, K. Fujii, K. Fukuya, K. Takashima, Influence of crystal orientation on hardness and nanoindentation deformation in ion-irradiated stainless steels, Journal of Nuclear Materials 417(1-3) (2011) 984-987.

[29] P. Dayal, D. Bhattacharyya, W.M. Mook, E.G. Fu, Y.Q. Wang, D.G. Carr, O. Anderoglu, N.A. Mara, A. Misra, R.P. Harrison, L. Edwards, Effect of double ion implantation and irradiation by Ar and He ions on nano-indentation hardness of metallic alloys, Journal of Nuclear Materials 438(1-3) (2013) 108-115.

[30] C. Xu, L. Zhang, W. Qian, J. Mei, X. Liu, The Studies of Irradiation Hardening of Stainless Steel Reactor Internals under Proton, Nuclear Engineering and Technology (2016) 1-7.

[31] A. Lupinacci, K. Chen, Y. Li, M. Kunz, Z. Jiao, G.S. Was, M.D. Abad, A.M. Minor, P. Hosemann, Characterization of ion beam irradiated 304 stainless steel utilizing nanoindentation and Laue microdiffraction, Journal of Nuclear Materials 458 (2015) 70-76.

[32] H. Oka, Y. Sato, N. Hashimoto, S. Ohnuki, Evaluation of multi-layered hardness in ionirradiated stainless steel by nano-indentation technique, Journal of Nuclear Materials 462 (2015) 470-474.

[33] W.D. Nix, H.J. Gao, Indentation size effects in crystalline materials: A law for strain gradient plasticity, Journal of the Mechanics and Physics of Solids 46(3) (1998) 411-425.

[34] Y. Huang, F. Zhang, K.C. Hwang, W.D. Nix, G.M. Pharr, G. Feng, A model of size effects in nano-indentation, Journal of the Mechanics and Physics of Solids 54(8) (2006) 1668-1686.

[35] I. Manika, J. Maniks, Effect of substrate hardness and film structure on indentation depth criteria for film hardness testing, Journal of Physics D-Applied Physics 41(7) (2008).

[36] R. Kasada, Y. Takayama, K. Yabuuchi, A. Kimura, A new approach to evaluate irradiation hardening of ion-irradiated ferritic alloys by nano-indentation techniques, Fusion Engineering and Design 86(9-11) (2011) 2658-2661.

[37] P.P. Liu, F.R. Wan, Q. Zhan, A model to evaluate the nano-indentation hardness of ionirradiated materials, Nuclear Instruments \& Methods in Physics Research Section B-Beam Interactions with Materials and Atoms 342 (2015) 13-18. 
[38] A.J.E. Foreman, M.J. Makin, Dislocation movement through random arrays of obstacles, Canadian Journal of Physics 45(2P2) (1967) 511-517.

[39] A.L. Gurson, Continuum theory of ductile rupture by void nucleation and growth. 1. Yeild critieria and flow rules for porous ductile media, Journal of Engineering Materials and Technology-Transactions of the Asme 99(1) (1977) 2-15.

[40] D. Tabor, A simple theory of static and dynamic hardness, Proceedings of the Royal Society of London Series a-Mathematical and Physical Sciences 192(1029) (1948) 247-274.

[41] M. Dao, N. Chollacoop, K.J. Van Vliet, T.A. Venkatesh, S. Suresh, Computational modeling of the forward and reverse problems in instrumented sharp indentation, Acta Materialia 49(19) (2001) 3899-3918.

[42] B.N. Singh, A.J.E. Foreman, H. Trinkaus, Radiation hardening revisited: role of intracascade clustering, Journal of Nuclear Materials 249(2-3) (1997) 103-115.

[43] X. Xiao, D. Song, J. Xue, H. Chu, H. Duan, A size-dependent tensorial plasticity model for FCC single crystal with irradiation, International Journal of Plasticity 65 (2015) 152-167.

[44] D. Kiener, P. Hosemann, S.A. Maloy, A.M. Minor, In situ nanocompression testing of irradiated copper, Nature Materials 10(8) (2011) 608-613.

[45] C.L. Woodcock, D.F. Bahr, Plastic zone evolution around small scale indentations, Scripta Materialia 43(9) (2000) 783-788.

[46] C.K. Dolph, D.J. da Silva, M.J. Swenson, J.P. Wharry, Plastic zone size for nanoindentation of irradiated Fe-9\%Cr ODS, Journal of Nuclear Materials 481 (2016) 33-45.

[47] H.F. Huang, D.H. Li, J.J. Li, R.D. Liu, G.H. Lei, S.X. He, Q. Huang, L. Yan, Nanostructure Variations and Their Effects on Mechanical Strength of Ni-17Mo-7Cr Alloy under Xenon Ion Irradiation, Materials Transactions 55(8) (2014) 1243-1247.

[48] K. Durst, B. Backes, M. Goken, Indentation size effect in metallic materials: Correcting for the size of the plastic zone, Scripta Materialia 52(11) (2005) 1093-1097.

[49] M. Liu, C. Lu, A.K. Tieu, Crystal plasticity finite element method modelling of indentation size effect, International Journal of Solids and Structures 54 (2015) 42-49.

[50] X. Xiao, D. Song, J. Xue, H. Chu, H. Duana, A self-consistent plasticity theory for modeling the thermo-mechanical properties of irradiated FCC metallic polycrystals, Journal of the Mechanics and Physics of Solids 78 (2015) 1-16.

[51] D. Song, X. Xiao, J. Xue, H. Chu, H. Duan, Mechanical properties of irradiated multi-phase polycrystalline BCC materials, Acta Mechanica Sinica 31(2) (2015) 191-204. 
[52] K. Farrell, T.S. Byun, N. Hashimoto, Deformation mode maps for tensile deformation of neutron-irradiated structural alloys, Journal of Nuclear Materials 335(3) (2004) 471-486. 